\title{
Symptoms related to mental disorder in healthcare workers during the COVID-19 pandemic in Brazil
}

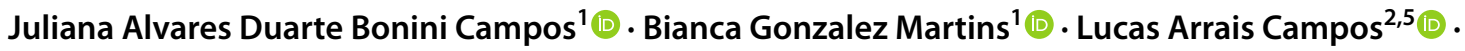 \\ Filomena de Fátima Valadão-Dias ${ }^{3}$ (1) . João Marôco ${ }^{4}$ (])
}

Received: 28 August 2020 / Accepted: 22 January 2021 / Published online: 9 February 2021

(c) The Author(s), under exclusive licence to Springer-Verlag GmbH, DE part of Springer Nature 2021

\begin{abstract}
Background Studies of previous pandemics indicate that healthcare workers have a high risk of developing symptoms related to mental health, especially depression, anxiety, and stress.

Objective To identify mental disorder symptoms among Brazilian healthcare workers during the Sars-Cov-2 pandemic and compare findings in different work categories.

Methods This was an online cross-sectional study. Information related to the pandemic and mental disorder symptoms was collected. The Depression, Anxiety, and Stress Scale and the Impact of Event Scale-revised were used. Associations were estimated by the chi-square test. The mean scores were compared among work categories with ANOVA $(\alpha=5 \%)$ and the prevalence of symptoms was estimated.

Results 1,609 healthcare workers participated in the survey [mean age: $36.9(\mathrm{SD}=11.6)$ years, women $=83.6 \%$ ]. There was no association between work category and changes in mental health during the pandemic $(p=0.288)$ or prevalence of unsafe feeling $(p=0.218)$. A significant relationship was observed between maintaining work activities during the pandemic and work category $(p<0.001)$. Physicians had the lowest out-of-work prevalence $(9.5 \%)$ while dentists had the highest $(32.3 \%)$. Physicians and nurses showed the highest prevalence of in-person work routine. Psychologists presented the highest prevalence of remote work (64.0\%) while dentists had the lowest (20.2\%). A high prevalence of depression (D), anxiety (A), and stress (S) symptoms was observed in all professional categories (D: 57.2, 95\% CI 48.3-66.1\%; A: 46.20\%, 95\% $\mathrm{CI}=37.2-55.2 \%$; S: 55.80\%, 95\% CI $=46.8-64.8 \%)$, with physicians $(\mathrm{D}=38.4 \%, \mathrm{~A}=25.80 \%, \mathrm{~S}=37.90 \%)$, psychologists $(\mathrm{D}=50.2 \%, \mathrm{~A}=39.0 \%, \mathrm{~S}=43.1 \%)$, and nurses $(\mathrm{D}=50.0 \%, \mathrm{~A}=40.9 \%, \mathrm{~S}=49.0 \%)$ having significantly lower scores. Psychologists had the lowest pandemic-related psychological impact (42.70\%, 95\% CI 36.8-48.6\%).

Conclusion Extreme changes in the work routine of dentists and psychologists and an overall high prevalence of mental symptoms due to the pandemic were found. Researchers should focus on gathering information that can identify workers at increased risk of mental illness to guide discussions and develop actions to minimize the harm of the pandemic. In addition, we suggest that healthcare and support systems urgently adopt mental health care measures with specialized professionals to protect the psychological well-being of the healthcare community.
\end{abstract}

Keywords Pandemic $\cdot$ Healthcare professionals $\cdot$ COVID-19 $\cdot$ Depression $\cdot$ Anxiety $\cdot$ Stress

\section{Introduction}

On March 11, 2020, the World Health Organization (WHO) declared the new coronavirus (Sars-Cov-2) disease (COVID19) a pandemic. At the end of that month in Brazil, due to the rapidly growing number of infected people, a mandatory

Juliana Alvares Duarte Bonini Campos

juliana.campos@unesp.br

Extended author information available on the last page of the article quarantine was decreed in the country for all except essential service personnel.

Due to drastic routine changes, need for fast adaptation to the new life style, and the unpredictability of events in a pandemic, people can show psychological reactions that could lead to emotional and social suffering (Brooks et al. 2020; Cullen et al. 2020; Huremovic 2019). Studies have reported a considerable increase in depression and anxiety symptoms and the development of post-traumatic stress due to the COVID-19 pandemic (Brooks et al. 2020; Cullen et al. 
2020; Huremovic 2019; Lai 2020; Pfefferbaum and North 2020; Usher et al. 2020; Wang et al. 2020). The psychological impact in healthcare workers is especially strong due to their increased risk of coronavirus infection, the fear of contaminating other people, particularly family members, insufficient availability of personal protective equipment and work settings protection, lack of operational protocols, and separation from family and friends (Ornell et al. 2020; Pfefferbaum and North 2020; Rajkumar 2020; Santamaría et al. 2020; Sethi et al. 2020; Shechter et al. 2020). Physicians, nurses, and dentists were found to have the highest risk of infection and transmission of the coronavirus, mainly due to the aerosol-generating procedures performed on patients (Occupational Safety and Health Administration 2020).

Peditto et al. (2020) and Coulthard (2020) report that the practice of Dentistry presents the highest risk of coronavirus infection among healthcare categories, which has been corroborated by other authors (Ahmed et al. 2020; Izzetti et al. 2020; Meng et al. 2020). This is due to the close interaction of dentists and dental staff with patients, frequent contact with saliva and blood, aerosol-generating procedures, and a high risk of cross-contamination from viruses in air particles, surfaces, and instruments. Some countries, such as Italy (Peditto et al. 2020) and China (Meng et al. 2020), have recommended the treatment of dental emergencies only, with elective treatments being suspended or postponed for after the control of the disease, which generates great impact on dentists' work routine.

Due to the ongoing pandemic and lack of data, studies related to the mental health of healthcare workers are increasing exponentially. Most of the publications are presented as letters to editors, brief reports, or in the case of Dentistry, presentation of protocols for adapted work routines. In July 2020, when the drafting of this text began, there were only five studies reporting data related to the subject: one from Spain (Santamaría et al. 2020), one from Pakistan (Sethi et al. 2020), two from China (Lai 2020; Zhang et al. 2020), and one with dentists from the Middle East (Ahmed et al. 2020). After a review in December 2020, a higher number of studies were found, including five systematic reviews (Luo et al. 2020; Muller et al. 2020; Pappa et al. 2020; Salazar de Pablo et al. 2020; Vizheh et al. 2020) that indicate high risk for mental problems of such workers, especially sleep disorders, depression, anxiety, stress, and overall psychological impact due to the pandemic. The studies that compared different work categories (Lai 2020; Luo et al. 2020; Pappa et al. 2020; Shechter et al. 2020; Vizheh et al. 2020) found that nurses had more symptoms than physicians. However, no study compared other categories of healthcare workers, whether from a hospital setting or otherwise.

Because of the high risk of psychological harm of healthcare workers during the pandemic, this study aimed to identify and compare psychological symptoms in different healthcare categories. The authors hope to provide initial evidence to raise awareness of the issue and support the development of measures for treatment and prevention.

\section{Methods}

\section{Study design and sample collection}

This was a cross-sectional study with a non-probabilistic snowball sampling method. Personal contacts of the authors that were healthcare workers were invited to participate by email or WhatsApp and were asked to pass on the invitation to their colleagues, including those from different regions of Brazil. A link to Google Forms containing the measurement instruments used in the research was included in the invitation message.

Participant information on work category, country region (Midwest, Northeast, North, Southeast, and South), age (years), sex, and average monthly family income $(<\mathrm{R} \$$ $1,255.00, \mathrm{R} \$ 1,255.00$ to $\mathrm{R} \$ 2,004.00, \mathrm{R} \$ 2,005.00$ to $\mathrm{R} \$$ $8,640.00, \mathrm{R} \$ 8,641.00$ to $\mathrm{R} \$ 11,261.00$ and $\geq \mathrm{R} \$ 11,262.00$ ) was collected, in addition to information related to the pandemic (Online Resource 1). Participants were also asked if they noticed a change in their mental health status after the start of the pandemic. Depression, anxiety, and stress symptoms and the psychological impact of the pandemic were assessed using the Depression, Anxiety and Stress Scale (DASS-21) (Lovibond and Lovibond 1995; Martins et al. 2019; Vignola and Tucci 2014) and the Impact of Event Scale-revised (IES-R) (Caiuby et al. 2012; Weiss and Marmar 1997).

\section{Procedures and ethical aspects}

Data collection was carried out online between May 18 and June 13, 2020 with those who agreed with the Informed Consent of the study. If a work category had less than 100 participants, the category was placed in the "other" category to maximize statistical power.

This study was approved by the National Research Ethics Commission of the Ministry of Health (CONEP) (CAAE 30604220.4.0000.0008) and therefore was performed in accordance with the ethical standards laid down in the 1964 Declaration of Helsinki and its later amendments.

\section{Measuring instruments}

The short version of the Depression, Anxiety and Stress Scale (DASS) has 21 items rated in a 4-point Likert-type response scale ( 0 : did not apply to me at all, 1 : some of the time, 2: a good part of time, 3: most of the time) (Lovibond 
and Lovibond 1995). The Portuguese version used was adapted from Vignola and Tucci (2014) by Martins et al. (2019).

To track the psychological impact of the pandemic, the Impact of Event Scale-revised (IES-R) (Weiss and Marmar 1997) was used. The Portuguese version was proposed by Caiuby et al. (2012). The IES-R has 22 items distributed in 3 factors (avoidance, intrusion, and hyperarousal) that can be evaluated separately or together (to assess general psychological impact). Responses are given in a 5-point Likert-type scale (0: not at all, 1: slightly, 2: moderately, 3 : very, and 4: extremely).

These instruments were previously used in a study by our research group and the data obtained with them presented adequate validity and reliability in a Brazilian sample (Campos et al. 2020). However, considering that validity and reliability are properties of the data and not of the instruments, the estimates of data validity and reliability are presented to support the quality of the study evidence.

\section{Data validity and reliability}

The fit of the models to the data was verified to ensure the validity and reliability of the data. The questionnaires' responses showed multivariate normality (Mardia's test <3.0). Confirmatory factor analysis (CFA) was performed using the weighted least squares means and variance adjusted (WLSMV). The DASS and IES-R goodness of the fit were assessed using the Comparative Fit Index (CFI), Tucker-Lewis Index (TLI), and Root Mean Square Error of Approximation (RMSEA). The fit was considered adequate if CFI and TLI $\geq 0.90$, and RMSEA $\leq 0.10$ (Kline 1998). The factorial loadings of the items were evaluated and considered satisfactory if $\lambda \geq 0.30$. Reliability was analyzed with the alpha ordinal coefficient $(\alpha)$ and considered adequate if $\alpha \geq 0.70$ (Table 1).

The fit of the models to the data was considered adequate, indicating the data validity and reliability. However, the DASS-21 model had no configurational invariance between physiotherapists and the other categories, limiting the direct comparison of the data. Regarding IES-R, there was no configurational invariance between physiotherapists and pharmacists and the other categories.

To carry out mean comparisons, the metric and scale invariances (Nolte and Elsworth 2014) were tested with delta CFI $(\triangle \mathrm{CFI})$ for the factorial weights, thresholds, and variances/covariance of the residuals. Values of $\Delta \mathrm{CFI}<-0.01$ were indicative of lack of model invariance. For the DASS21 , the invariance was tested without including physiotherapists and a strong measurement invariance $(\Delta \mathrm{CFI}=-0.001$
Table 1 Psychometric parameters indicating the goodness-of-fit to the data of the DASS (Depression, Anxiety and Stress Scale) and IES-R models (Impact of Event Scalerevised)

\begin{tabular}{|c|c|c|c|c|c|c|c|}
\hline \multirow[t]{2}{*}{ Instrument } & \multirow[t]{2}{*}{ Professional category } & \multirow[t]{2}{*}{$n$} & \multicolumn{4}{|l|}{$\mathrm{CFA}^{\mathrm{a}}$} & \multirow[t]{2}{*}{$\alpha$} \\
\hline & & & $\lambda$ & CFI & TLI & RMSEA [90\%CI] & \\
\hline \multirow[t]{8}{*}{ DASS-21 } & Dentist & 341 & $0.55-0.91$ & 0.985 & 0.983 & $0.053[0.045-0.061]$ & $0.877-0.945$ \\
\hline & Nurse & 198 & $0.50-0.91$ & 0.977 & 0.974 & $0.058[0.047-0.069]$ & $0.887-0.933$ \\
\hline & Pharmacist & 157 & $0.55-0.89$ & 0.966 & 0.962 & $0.070[0.057-0.082]$ & $0.872-0.930$ \\
\hline & Physiotherapist $^{\mathrm{b}}$ & 115 & $0.50-0.97$ & 0.977 & 0.973 & $0.068[0.051-0.084]$ & $0.884-0.938$ \\
\hline & Physician & 190 & $0.46-0.90$ & 0.972 & 0.968 & $0.060[0.048-0.071]$ & $0.876-0.937$ \\
\hline & Nutritionist & 133 & $0.47-0.92$ & 0.950 & 0.944 & $0.077[0.063-0.090]$ & $0.890-0.915$ \\
\hline & Psychologist & 267 & $0.58-0.93$ & 0.974 & 0.971 & $0.063[0.054-0.072]$ & $0.886-0.944$ \\
\hline & Others & 208 & $0.50-0.95$ & 0.974 & 0.971 & $0.075[0.065-0.085]$ & $0.878-0.945$ \\
\hline \multirow[t]{8}{*}{ IES-R } & Dentist $^{c}$ & 341 & $0.40-0.90$ & 0.953 & 0.947 & $0.083[0.076-0.091]$ & $0.869-0.928$ \\
\hline & Nurse $^{c}$ & 198 & $0.53-0.91$ & 0.981 & 0.978 & $0.055[0.043-0.067]$ & $0.858-0.925$ \\
\hline & Pharmacist $^{\mathrm{d}}$ & 157 & $0.46-0.89$ & 0.954 & 0.947 & $0.087[0.074-0.100]$ & $0.867-0.900$ \\
\hline & Physiotherapist $^{\mathrm{e}}$ & 115 & $0.45-0.92$ & 0.961 & 0.957 & $0.076[0.061-0.090]$ & $0.878-0.932$ \\
\hline & Physician $^{\mathrm{c}}$ & 190 & $0.30-0.92$ & 0.973 & 0.969 & $0.058[0.046-0.069]$ & $0.844-0.906$ \\
\hline & Nutritionist $\mathrm{c}^{\mathrm{c}}$ & 133 & $0.36-0.94$ & 0.962 & 0.957 & $0.072[0.058-0.085]$ & $0.840-0.928$ \\
\hline & Psychologist $^{\mathrm{c}}$ & 267 & $0.51-0.86$ & 0.963 & 0.958 & $0.065[0.056-0.073]$ & $0.843-0.917$ \\
\hline & Others $^{\mathrm{c}}$ & 208 & $0.40-0.91$ & 0.955 & 0.949 & $0.076[0.066-0.086]$ & $0.836-0.913$ \\
\hline
\end{tabular}

${ }^{a}$ Confirmatory factor analysis by weighted least squares mean and variance adjusted (WLSMV), comparative fit index (CFI), Tucker-Lewis index (TLI), and root mean square error of approximation (RMSEA) estimation with $90 \%$ confidence interval $[90 \% \mathrm{CI}]$. Adequate fit: CFI and TLI $\geq 0.90$, RMSEA $\leq 0.10$, and $\alpha>0.70$

${ }^{\mathrm{b}}$ Refined model without item 15

${ }^{\mathrm{c}}$ Refined model without item 2

${ }^{\mathrm{d}}$ Refined model without items 2, 5, and 7

${ }^{\mathrm{e}}$ Full model 
to - 0.003) was found. For IES-R, pharmacists and physiotherapists were not included in the CFI analysis. No invariance was found for IES-R of physicians in relation to the others $(\Delta \mathrm{CFI}<-0.01 ; p<0.001)$, which may have occurred due to responses being concentrated at the lower end of the scale differently from the other work categories (Fig. 1); therefore, mean comparisons did not include physicians. The MPLUS 7.2 program (Muthén and Muthén, Los Angeles, CA) was used for the above analyses.

\section{Data analysis}

The association between work category and the variables mental health change (measured by the question: "Did you notice any change in your mental health status after the start of the pandemic?"), feeling of unsafe, and work factors during the pandemic was estimated using the chi-square test. Multiple comparisons were performed by analysis of standardized residuals ( $z$ test). The comparison of depression, anxiety, and stress mean scores and psychological impact (general, avoidance, intrusion, and hyperarousal) between work categories was performed using ANOVA. The assumption of normality was met $(p>0.05)$. Multiple comparisons were performed with the Tukey's post-test when homoscedasticity (Levene's test) was present, otherwise, the Welch's ANOVA and Games-Howell post-test $(\alpha=5 \%)$ were used.

The symptoms' prevalence in different work categories was estimated by a $95 \%$ confidence interval (95\% CI) using the cutoffs proposed by Lovibond and Lovibond (1995) (sum of scores for Depression: absent $\leq 9$; present $\geq 10$; Anxiety: absent $\leq 7$; present $\geq 8$; Stress: absent $\leq 14$; present $\geq 15$ ) and by Wang et al. (2020) (Psychological impact: Normal/
Fig. 1 Mean scores for the responses to the items of the Depression, Anxiety and Stress Scale (DASS-21) and the Impact of Event Scale—revised (IES-R) according to work category

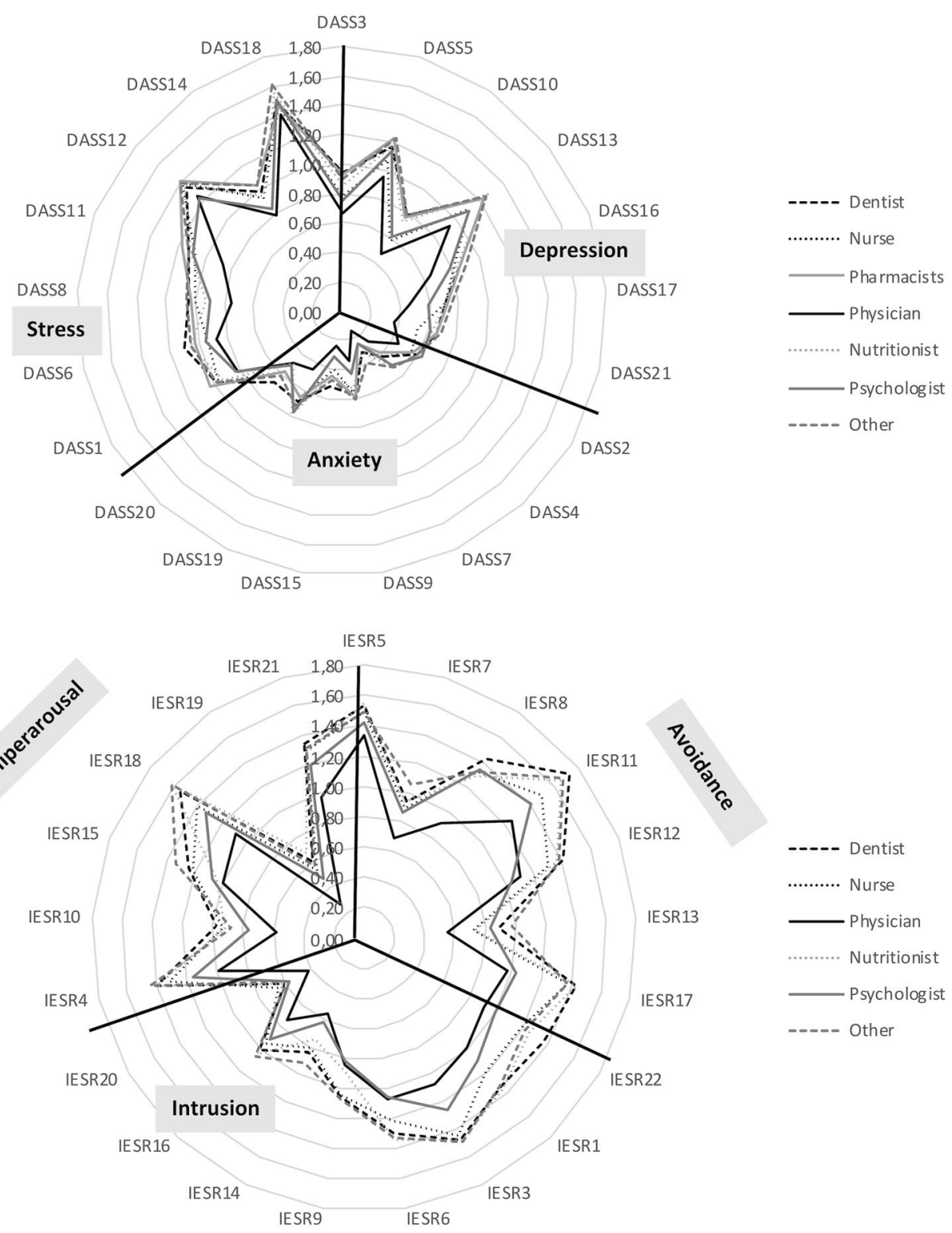


absent $<23$; present $\geq 24$ ). The IBM SPSS Statistics program (v. 22, IBM Corp, Armonk, NY) was used for data analyses.

\section{Results}

A total of 1,609 healthcare workers [mean age $=36.9$ (standard deviation $=11.6$ ) years] participated in the study (Table 2).

The majority $(62.9 \%)$ reported feeling unsafe, $21.7 \%$, very unsafe, $14.7 \%$, safe, and $0.7 \%$, very safe, independent of work category $\left.\left[X^{2}(21)=9,506 ; p=0.218\right]\right)$. During the pandemic period, $90.3 \%$ reported symptoms related to mental disorder. Among individuals who had no previous symptom, $88 \%$ started having symptoms of psychological illness after the start of the pandemic. Of the total, $69.1 \%$ noticed changes in their mental health with no significant association with work category $\left[X^{2}(21)=8.536 ; p=0.288\right]$.

A significant association was found between job factors and work category $\left[X^{2}(21)=379.7 ; p<0.001\right]$ (Table 3). Compared to the other categories, fewer physicians were out of work due to the pandemic $(z=-3.6 ; p<0.001)$ and dentists had the highest prevalence of workers staying at home $(z=4.3 ; p<0.001)$. Workers that most kept the usual work routine (in-person work) were physicians $(z=3.7$; $p<0.001)$ and nurses $(z=5.9 ; p<0.001)$ while dentists $(z=-4.8 ; p<0.001)$, nutritionists $(z=-2.1)$, and psychologists $(z=-3.6 ; p<0.001)$ were more often out of the usual routine. Physicians $(z=7.1 ; p<0.001)$ and dentists $(z=5.1$; $p<0.001$ ) were those who most required work routine adaptation. The highest prevalence of remote work was observed among psychologists $(z=7.5 ; p<0.001)$. The prevalence of remote work was also significantly higher among nutritionists $(z=2.1 ; p=0.036)$ and pharmacists $(z=2.9 ; p=0.004)$. With regard to psychological symptoms, anxiety had the lowest scores compared to the depression and stress.

Physicians, psychologists, and nurses had significantly lower scores for depression and anxiety than the other categories. Overall, the psychological impact of the pandemic was lower among psychologists (Table 4). Figure 1 shows the mean scores of each item in the DASS-21 and the IES-R scales. The lowest scores of items related to the Anxiety factor (DASS-21) should raise attention.

A high prevalence of depression, anxiety, and stress symptoms, as well as psychological impact due to the pandemic was found in all work categories (Table 5), except in physicians, which was a remarkable finding.

\section{Discussion}

As known, the effects of pandemics are reflected in feelings, behaviors, and emotional responses of individuals and in the community as a whole (Huremovic 2019), and
Table 2 Characteristics of the healthcare workers participating in the study $(n=1,609)$

\begin{tabular}{|c|c|}
\hline Characteristic & $n(\%)$ \\
\hline \multicolumn{2}{|c|}{ Previous diagnosis of mental disorder } \\
\hline No & $1,121(69.7)$ \\
\hline Yes & $448(27.8)$ \\
\hline \multicolumn{2}{|c|}{ Know someone who tested positive for COVID-19 } \\
\hline No & $380(23.6)$ \\
\hline Yes & $1,229(76.4)$ \\
\hline \multicolumn{2}{|l|}{ Social isolation } \\
\hline No & $349(21.7)$ \\
\hline Yes & $1,260(78.3)$ \\
\hline \multicolumn{2}{|c|}{ Believe the COVID-19 is dangerous } \\
\hline No & $36(2.2)$ \\
\hline Yes & $1,573(97.8)$ \\
\hline \multicolumn{2}{|l|}{ Monthly family income $^{a}$} \\
\hline$<\mathrm{R} \$ 1,255.00$ & $48(3.0)$ \\
\hline $\mathrm{R} \$ 1,255.00-\mathrm{R} \$ 2,004.00$ & $117(7.3)$ \\
\hline $\mathrm{R} \$ 2,005.00-\mathrm{R} \$ 8,640.00$ & $711(44.2)$ \\
\hline $\mathrm{R} \$ 8,641.00-\mathrm{R} \$ 11,261.00$ & $273(17.0)$ \\
\hline$>\mathrm{R} \$ 11,262.00$ & $457(28.5)$ \\
\hline \multicolumn{2}{|l|}{ Previous health problem } \\
\hline No & $1,182(73.5)$ \\
\hline Yes & $427(26.5)$ \\
\hline \multicolumn{2}{|l|}{ Gender } \\
\hline Women & $1,338(83.6)$ \\
\hline Men & $263(16.4)$ \\
\hline \multicolumn{2}{|l|}{ Region in Brazil } \\
\hline Midwest & $91(5.7)$ \\
\hline Northeast & $479(27.8)$ \\
\hline North & $142(8.8)$ \\
\hline Southeast & $702(43.6)$ \\
\hline South & $195(12.1)$ \\
\hline \multicolumn{2}{|l|}{ Professional category } \\
\hline Dentist & $341(21.2)$ \\
\hline Nurse & $198(12.3)$ \\
\hline Pharmacists & $157(9.8)$ \\
\hline Physiotherapist & $115(7.1)$ \\
\hline Physician & $190(11.8)$ \\
\hline Nutritionist & $133(8.3)$ \\
\hline Psychologist & $267(16.6)$ \\
\hline Other $^{\mathrm{b}}$ & 208 (12.9) \\
\hline
\end{tabular}

${ }^{\mathrm{a}} 1 \mathrm{USD}=5.24 \mathrm{BRL}(\mathrm{R} \$)(12 / 02 / 2020)$

bealth agent: $n=22$, biomedical agent: $n=55$, physical educator: $n=11$, speech therapist: $n=42$, health technician: $n=48$, and occupational therapist: $n=30$

healthcare workers are at increased risk for psychological harm (Ahmed et al. 2020; Barabari and Moharamzadeh 2020; Brooks et al. 2020; Grover et al. 2020; Lai 2020; Moreno et al. 2020; Neto et al. 2020; Ornell et al. 2020; Santamaría et al. 2020; Sethi et al. 2020). This study 
Table 3 Distribution of participants according to work category and work characteristics during the pandemic

\begin{tabular}{lccccc}
\hline Professional category & \multicolumn{2}{l}{$n(\%)$ Are you still working during the pandemic? } & Total \\
\cline { 2 - 5 } & No & $\begin{array}{c}\text { Yes, usual rou- } \\
\text { tine, in-person }\end{array}$ & $\begin{array}{l}\text { Yes, adapted in- } \\
\text { person routine }\end{array}$ & Yes, remotely & \\
\hline Dentist & $110(32.3)$ & $13(3.8)$ & $149(43.7)$ & $69(20.2)$ & 341 \\
Nurse & $42(21.2)$ & $57(28.8)$ & $32(16.2)$ & $67(33.8)$ & 198 \\
Pharmacists & $24(15.3)$ & $24(15.3)$ & $30(19.1)$ & $79(50.3)$ & 157 \\
Physiotherapist & $28(24.4)$ & $16(13.9)$ & $32(27.8)$ & $39(33.9)$ & 115 \\
Physician & $18(9.5)$ & $44(23.2)$ & $107(56.3)$ & $21(11.0)$ & 190 \\
Nutritionist & $37(27.8)$ & $9(6.8)$ & $24(18.0)$ & $63(47.4)$ & 133 \\
Psychologist & $41(15.4)$ & $14(5.2)$ & $41(15.4)$ & $171(64.0)$ & 267 \\
Other & $47(22.6)$ & $38(18.2)$ & $49(23.6)$ & $74(35.6)$ & 208 \\
\hline
\end{tabular}

Table 4 Comparison of the mean scores for depression, anxiety and stress symptoms and the psychological impact related to the pandemic (general, avoidance, intrusion and hyperarousal) among work categories

\begin{tabular}{|c|c|c|c|c|c|c|c|}
\hline \multirow[t]{3}{*}{ Professional category } & \multicolumn{7}{|c|}{ Mean (standard deviation) } \\
\hline & \multicolumn{3}{|l|}{ DASS- $21 *$} & \multicolumn{4}{|l|}{ IES-R* } \\
\hline & Depression $^{\#}$ & Anxiety $^{\#}$ & Stress $^{\mathfrak{E}}$ & Psychologic impact $^{£}$ & Avoidance $^{£}$ & Intrusion $^{\mathfrak{f}}$ & Hyperarousal $^{\#}$ \\
\hline Dentist & $12.7(9.7)^{\mathrm{b}}$ & $7.6(6.9)^{\mathrm{b}}$ & $16.1(8.4)^{\mathrm{b}}$ & $26.8(15.6)^{\mathrm{b}}$ & $10.8(6.1)^{\mathrm{b}}$ & $8.9(6.5)^{b}$ & $7.1(5.1)^{\mathrm{b}}$ \\
\hline Nurse & $11.2(9.0)^{\mathrm{a}, \mathrm{b}}$ & $6.9(6.8)^{\mathrm{b}}$ & $15.5(7.9)^{\mathrm{a}, \mathrm{b}}$ & $25.0(15.0)^{\mathrm{a}, \mathrm{b}}$ & $9.8(6.0)^{\mathrm{a}, \mathrm{b}}$ & $8.5(6.1)^{\mathrm{a}, \mathrm{b}}$ & $6.7(4.7)^{\mathrm{a}, \mathrm{b}}$ \\
\hline Pharmacist ${ }^{\mathfrak{f}}$ & $12.8(9.4)^{\mathrm{b}}$ & $6.9(6.8)^{b}$ & $16.3(8.6)^{b}$ & $27.0(13.7)$ & $10.6(5.5)$ & $9.2(6.0)$ & $7.2(4.8)$ \\
\hline Physician ${ }^{£ f £}$ & $9.0(8.2)^{\mathrm{a}}$ & $4.7(5.4)^{\mathrm{a}}$ & $13.5(7.2)^{\mathrm{a}}$ & $19.3(12.0)$ & $7.7(5.5)$ & $6.7(4.7)$ & $4.9(3.7)$ \\
\hline Nutritionist & $12.4(8.8)^{\mathrm{b}}$ & $7.4(7.0)^{\mathrm{b}}$ & $15.7(8.5)^{\mathrm{a}, \mathrm{b}}$ & $25.8(14.7)^{\mathrm{a}, \mathrm{b}}$ & $10.2(5.5)^{\mathrm{a}, \mathrm{b}}$ & $8.7(6.4)^{\mathrm{a}, \mathrm{b}}$ & $7.0(5.0)^{\mathrm{a}, \mathrm{b}}$ \\
\hline Psychologist & $11.2(9.4)^{\mathrm{ab}}$ & $6.8(6.7)^{\mathrm{b}}$ & $14.7(7.9)^{\mathrm{a}, \mathrm{b}}$ & $22.4(13.2)^{\mathrm{a}}$ & $8.9(5.8)^{\mathrm{a}}$ & $7.5(5.7)^{\mathrm{a}}$ & $6.0(4.3)^{\mathrm{a}}$ \\
\hline Others & $13.1(10.1)^{\mathrm{b}}$ & $7.8(7.0)^{\mathrm{b}}$ & $16.5(8.6)^{b}$ & $26.7(14.5)^{b}$ & $10.5(5.4)^{\mathrm{b}}$ & $9.0(6.1)^{\mathrm{b}}$ & $7.2(5.0)^{\mathrm{a}, \mathrm{b}}$ \\
\hline
\end{tabular}

*DASS-21: Depression, Anxiety and Stress Scale, IES-R: Impact of Event Scale-revised

\#Welch corrected ANOVA (Games-Howell post-test)

${ }^{£}$ ANOVA (Tukey's post-test); ab, different letters indicate statistical difference, $\alpha=5 \%$

${ }^{£ \mathfrak{P}}$ Pharmacist category was not included in the comparison of IES-R scores due to lack of configurational invariance

${ }^{\mathfrak{f} \mathrm{f}}$ Physician category was not included in the comparison of IES-R scores due to lack of metric and scalar invariance

Table 5 Prevalence (point estimate and 95\% confidence interval-95\% CI) of depression, anxiety, stress, and general psychological impact according to work category

\begin{tabular}{llllr}
\hline & & & & \\
\cline { 2 - 5 } Professional category & Depression & Anxiety & Stress & Overall Psychologic impact \\
\hline Dentist & $57.2[51.9-62.5]$ & $43.10[37.8-48.4]$ & $49.90[44.6-55.2]$ & $50.40[45.1-55.7]$ \\
Nurse & $50.0[43.0-57.0]$ & $40.90[34.0-47.8]$ & $49.00[42.0-56.0]$ & $46.00[39.0-53.0]$ \\
Pharmacist & $59.2[51.5-66.9]$ & $40.10[32.4-47.8]$ & $54.10[46.3-61.9]$ & $55.4[47.6-63.2]$ \\
Physician & $38.4[31.5-45.3]$ & $25.80[19.6-32.0]$ & $37.90[31.0-44.8]$ & $31.6[25.0-38.2]$ \\
Nutritionist & $55.6[47.1-64.1]$ & $40.60[32.2-49.0]$ & $51.90[43.4-60.4]$ & $51.1[42.6-59.6]$ \\
Psychologist & $50.2[44.2-56.2]$ & $39.00[33.1-44.9]$ & $43.10[37.1-49.1]$ & $42.70[36.8-48.6]$ \\
Other & $57.2[48.3-66.1]$ & $46.20[37.2-55.2]$ & $55.80[46.8-64.8]$ & $54.80[45.8-63.8]$ \\
\hline
\end{tabular}

presents information on immediate symptoms of mental disorder in healthcare workers during the Sars-Cov-2 pandemic and a comparison of symptoms among work categories. Changes in work routine, especially for dentists and psychologists, and a high prevalence of depression, anxiety, and stress symptoms and overall psychological impact of the pandemic were observed in all work categories. The validity and reliability of the data were tested and affirmed, supporting the quality of the evidence presented. 
As previously mentioned, dentists are at the highest risk for coronavirus infection (Ahmed et al. 2020; Barabari and Moharamzadeh 2020; Meng et al. 2020; Peditto et al. 2020) due to the nature of dental procedures, which is probably the main cause of the high proportion of dentists (32.3\%) that have stopped their activities. In addition, the little information regarding the virus mechanisms of action and the disease's prevention and treatment, added to the lack a highly efficient dental care protocol might have contributed to the decision of stopping work activities. Moreover, since the beginning of the pandemic, dentists were advised by local authorities to provide only emergency dental care, seriously affecting regular activities, especially in private offices that provide mostly elective treatments. Thus, the COVID-19 pandemic has caused immediate and possibly medium- and long-term implications for Dentistry (Barabari and Moharamzadeh 2020; Izzetti et al. 2020). In the short term, suspension of practice will certainly result in financial loss (Sethi et al. 2020) and the need to develop strategies to return to normal activities. In the medium and long term, investments will be needed for the adaptation of clinical settings to the new scenario with optimization of infection control protocols, fewer daily appointments, and training and education of the dental staff and patients (Barabari and Moharamzadeh 2020; Izzetti et al. 2020). Work routine adaptation was reported by $43.7 \%$ of dentists.

On the other hand, most physicians and nurses continued working, which was expected as they are at the front line of the pandemic. However, more than half of the physicians reported adapting the work routine to the new reality (56.3\%). Following recommendations from the Federal Council of Psychology to maintain clinical care during the pandemic, the majority of phycologists were working but over $60 \%$ were providing care remotely. Psychological support is essential for the population in general and specifically for those with previous mental health problems, as the current context of isolation, quarantine, restriction of movement, and routine alteration increases the overall risk of psychological symptoms (Grover et al. 2020; Huremovic 2019; Moreno et al. 2020; Pfefferbaum and North 2020; Usher et al. 2020). Unfortunately, remote work is impossible for other healthcare workers, especially physicians and dentists, as most clinical procedures of such categories are provided in person, increasing the exposure of these professionals and their risk of physical and mental harm. The sudden and extreme changes in work routines also add to the increased risk of psychological symptoms as reported in previous studies (Grover et al. 2020; Lai 2020; Ornell et al. 2020; Santamaría et al. 2020; Sethi et al. 2020).

The prevalence of depression, anxiety, and stress symptoms observed among the categories was higher than those reported by a Spanish study $(n=421)$ (Santamaría et al. 2020) and in the meta-analysis presented by Salazar de
Pablo et al. (2020). The political, economic, and social crises that are ongoing in Brazil simultaneously to the health crisis and a large amount of inaccurate and often conflicting information from the media and the government regarding the coronavirus and its prevention and treatment measures (Brooks et al. 2020; Usher et al. 2020) increase the feeling of unsafe, uncertainty, and lack of control, and can consequently cause psychological suffering. In a study from Jordan (Naser 2020), a high prevalence of depression and anxiety was also identified among healthcare workers $(n=1,163)$. Sethi et al. $(2020)$, in a qualitative study with Pakistani healthcare workers (physicians: $n=237$ and dentists: $n=35$ ), Shechter et al. (2020), in a New York hospital study (nurse: $n=313$ and physicians: $n=344$ ), and Salazar de Pablo (2020), in a systematic review reported an impact of the pandemic on mental (insomnia, psychological distress, anxiety, and depressive symptoms), physical (cough, fatigue, fever, headaches, and sore throat), social health, and well-being. Similar findings were also reported in other systematic reviews that include studies from China, Singapore, Italy, Iran, and Spain (Luo et al. 2020; Muller et al. 2020; Pappa et al. 2020; Vizheh et al. 2020). However, as far as we know, there are no data related to psychological symptoms from the pandemic in Brazilian healthcare workers that can be compared with the findings of the present study.

The lower prevalence of mental health symptoms among Brazilian physicians should be interpreted with caution. Ornell et al. (2020) advise that healthcare workers on the front line of the pandemic are viewed as superheroes, which on the one hand shows the appreciation from the population, and on the other hand, can add pressure to the already stressful routine of these workers as to not make mistakes, give up, or get sick. Low prevalence of these symptoms among physicians was also found by Lai (2020) in China and by Shechter et al. (2020) in the United States. Perhaps, the evaluated physicians were still highly focused on the emergency of the situation without yet having the time and space to develop and express their personal feelings. Although lower than the other categories, physicians still had depressive symptoms above normal, but they might be dealing with daily pandemic-related stressors by rescuing coping strategies developed in previous experiences. This could also explain the lower scores of depression and anxiety among physicians and nurses compared to other professionals. However, more studies are needed to verify this theory.

Although no significant difference was found in psychological symptoms between dentists and the other professional categories, a large proportion of dentists were out of work during the pandemic. Such situation might decrease stressors in the short term but may have important consequences in the medium and long term, either due to financial losses or to the drastic changes in future work settings. 
Therefore, the mental health of these professionals should be followed up.

Regarding the psychological impact of the pandemic, in general, psychologists had an overall lower score, which can be related to their training and better ability to develop adaptive strategies. However, this category was not immune to the deleterious effects of the pandemic on physical, mental, and social wellbeing, indicated by the high prevalence of depression, anxiety, and stress symptoms. Psychologists should be aware of their own symptoms and find the resources to maintain their own mental health.

An interesting finding of this study was the lower mean score for anxiety compared to stress, depression, and psychological impact of the pandemic (avoidance, intrusion and hyperarousal) (Fig. 1). These findings can be supported by the temporal orientation underlying psychological symptoms (Eysenck et al. 2006; Rinaldi et al. 2017). Within this theory, stress is related to present stimuli, anxiety, to future threatening events, and depression is related to losses in the past. In times of crisis and in high impact events, such as a pandemic, the event is immediately felt as a stressor and memories and previous experiences gain strength from the losses inherent to the transformations that could result from the pandemic, triggering depressive symptoms. Thus, the consequences and losses related to the event occur in the present, which are managed by rescuing past coping strategies, with no mental availability for thinking and reacting to an uncertain future. This mental process, however, could change with the course of the pandemic and increase the anxiety levels. Thus, healthcare workers should be monitored to minimize the occurrence of anxiety that may arise with the progress of events related to the pandemic and the subsequent uncertainties that will occur over time.

Despite the important findings and originality of this study, some limitations need to be reported. First, only overall mental health symptoms were assessed in the participants without exploring in-depth the work activities performed before and during the pandemic. However, this was done to rapidly present information on the mental health of this population and foster an immediate discussion about the impact of the pandemic and provide the needed support, since in Brazil, this information was not yet available. In addition, using an online data collection method, we prioritized the inclusion of the largest possible number of workers from different areas and a short time for completion of questionnaires to optimize compliance. Based on our findings, further studies can now be carried out with additional data related to participants' work hours, specific work fields, type of position (private, public, tenured), and being or not on the front line of the pandemic.

Another limitation is the use of non-probabilistic sampling and online data collection, which may have limited the number of participants and hindered generalizability of the data. The non-probabilistic sampling design also does not allow us to identify the adherence rate of health workers to the study since there is no way to know how many invitations were sent out. Pierce et al. (2020) states that this study design in the mental health field may underestimate the prevalence of mental disorders since people seriously affected would scarcely participate in the study. Thus, the estimated levels of psychological symptoms should be interpreted with caution because the willingness to participate can vary based on the level of distress, jeopardizing the representativeness of the sample. However, this strengthens the importance of our findings as the prevalence of mental health symptoms may be even higher than found. Our study could be considered a starting point for further investigations with more in-depth data, more appropriate designs, and long-term follow-up for developing actions aimed at supporting healthcare workers.

The psychological/psychiatric needs of healthcare workers should not be neglected during and after the pandemic. Researchers should focus on gathering information that can identify workers at increased risk of mental illness to guide discussions and develop actions to minimize the harm of the pandemic. In addition, we suggest that healthcare and support systems urgently adopt mental health care measures with specialized professionals to protect the psychological well-being of the healthcare community. Ornell et al. (2020) suggest that the recognition of risks and the identification of a history of psychosocial exposure should be a priority, which can be done, for example, using tracking instruments and offering a place for listening. Also, strategies for psychological intervention and coping must be planned, such as intra- and inter-team support and communication systems, mutual empathy and understanding, and the establishment of a space for the expression of feelings and symptoms, such as exhaustion and helplessness. These strategies can be implemented face-to-face or remotely, and may include counseling, crisis management interventions, monitoring and treatment of symptoms, lifestyle guidance, stress management, and pharmacological treatment of severe cases. The authors also suggest the involvement of healthcare managers for the development of guidelines and policies aimed at maintaining mental health and overall well-being of healthcare workers.

\section{Conclusion}

Dentists and psychologists were the workers who most changed their work routine. A high prevalence of depression, anxiety, and stress symptoms and psychological impact was observed in all healthcare work categories. Support strategies must be adopted immediately and in the long term to 
minimize the harmful effects of the pandemic on the mental health of this population.

Supplementary Information The online version contains supplementary material available at https://doi.org/10.1007/s00420-021-01656-4.

Acknowledgements We thank the study participants and Beatriz Buda Fuller for her assistance in data collection. This study was financed in part by the Coordenação de Aperfeiçoamento de Pessoal de Nível Superior-Brasil (CAPES)—Finance Code 001. We also thank the São Paulo Research Foundation (FAPESP) (Grant \#2020/08239-6).

Author contributions JADBC: contributed to conception and design, data acquisition, analysis, and interpretation, drafted the manuscript. BGM: contributed to conception and design, data acquisition, analysis, and interpretation, critically revised the manuscript. LAC: contributed to conception and design, data acquisition, analysis, and interpretation, critically revised the manuscript. FFVD: contributed to data interpretation, critically revised the manuscript. JM: contributed to design, data interpretation, critically revised the manuscript. All authors gave their final approval and agree to be accountable for all aspects of the work.

Funding This study was financed in part by the Coordenação de Aperfeiçoamento de Pessoal de Nível Superior-Brasil (CAPES) - Finance Code 001 and by the São Paulo Research Foundation (FAPESP) (Grant \#2020/08239-6).

\section{Compliance with ethical standards}

Conflict of interest The authors declare that they have no conflict of interest.

Ethics approval This study was approved by the National Research Ethics Commission of the Ministry of Health (CONEP) (CAAE 30604220.4.0000.0008) and therefore was performed in accordance with the ethical standards laid down in the 1964 Declaration of Helsinki and its later amendments.

\section{References}

Ahmed MA, Jouhar R, Ahmed N, Adnan S, Aftab M, Zafar MS, Khurshid Z (2020) Fear and practice modifications among dentists to combat novel coronavirus disease (COVID-19) outbreak. Int J Environ Res Public Health 17:e2821. https://doi.org/10.3390/ijerp h17082821

Barabari P, Moharamzadeh K (2020) Novel coronavirus (COVID19) and dentistry - a comprehensive review of literature. Dent J 8:1-18. https://doi.org/10.3390/dj8020053

Brooks SK, Webster RW, Smith LE, Woodland L, Wessely S, Greenberg N, Rubin GJ (2020) The psychological impact of quarantine and how to reduce it: rapid review of the evidence. Lancet 395:912-920. https://doi.org/10.1016/S0140-6736(20)30460-8

Caiuby AVS, Lacerda SS, Quintana MI, Torii TS, Andreoli SB (2012) Cross-cultural adaptation of the Brazilian version of the impact of events scale-revised (IES-R). Cad Saude Pública 28:597-603. https://doi.org/10.1590/s0102-311x2012000300019

Campos JADB, Martins BG, Campos LA, Maroco J, Saadiq RA, Ruano R (2020) Early psychological impact of the COVID-19 pandemic in Brazil: a national survey. J Clin Med 9:2976. https:// doi.org/10.3390/jcm9092976
Coulthard P (2020) Dentistry and coronavirus (COVID-19)—moral decision-making. Br Dent J 228:503-505. https://doi.org/10.1038/ s41415-020-1482-1

Cullen W, Gulati G, Kelly BD (2020) Mental health in the Covid19 pandemic. QJM 113:311-312. https://doi.org/10.1093/qjmed /hcaa110

Eysenck MW, Payne S, Santos R (2006) Anxiety and depression: past, present, and future events. Cogn Emot 20:274-294. https://doi. org/10.1080/02699930500220066

Grover S, Dua D, Sahoo S, Mehra A, Nehra R, Chakrabart S (2020) Why all COVID-19 hospitals should have mental health professionals: the importance of mental health in a worldwide crisis. Asian J Psychiatr 51:e102147. https://doi.org/10.1016/j. ajp.2020.102147

Huremovic D (2019) Psychiatry of pandemics. A mental health response to infection outbreak, Springer Nature, Switzerland

Izzetti R, Nisi M, Gabriele M, Graziani F (2020) COVID-19 transmission in dental practice: brief review of preventive measures in Italy. J Dent Res 99:1030-1038. https://doi.org/10.1177/00220 34520920580

Kline RB (1998) Principles and practice of structural equation modeling. The Guilford Press, New York

Lai J et al (2020) Factors associated with mental health outcomes among health care workers exposed to coronavirus disease 2019 . JAMA Netw Open 3:203976. https://doi.org/10.1001/jamanetwor kopen.2020.3976

Lovibond SH, Lovibond PF (1995) Manual for the depression, anxiety, stress scales. Psychology Foundation, Sydney

Luo M, Guo L, Yu M, Jiang W, Wang H (2020) The psychological and mental impact of coronavirus disease 2019 (COVID-19) on medical staff and general public - a systematic review and metaanalysis. Psychiatry Res 291:113190. https://doi.org/10.1016/j. psychres.2020.113190

Martins BG, Silva WR, Maroco J, Campos JADB (2019) Depression, anxiety, and stress scale: psychometric properties and affectivity prevalence. J Bras Psiquiatr 68:32-41. https://doi. org/10.1590/0047-2085000000222

Meng L, Hua F, Bian Z (2020) Coronavirus disease 2019 (COVID-19): emerging and future challenges for dental and oral medicine. $\mathrm{J}$ Dent Res 99:481-487. https://doi.org/10.1177/002203452091424 6

Moreno C et al (2020) How mental health care should change as a consequence of the COVID-19 pandemic. Lancet Psychiatry 7:813-824. https://doi.org/10.1016/S2215-0366(20)30307-2

Muller AE et al (2020) The mental health impact of the covid-19 pandemic on healthcare workers, and interventions to help them: a rapid systematic review. Psychiatry Res 293:113441. https://doi. org/10.1016/j.psychres.2020.113441

Naser AY et al (2020) Mental health status of the general population, healthcare professionals, and university students during 2019 coronavirus disease outbreak in Jordan: a cross-sectional study. Brain Behav 10:1730. https://doi.org/10.1002/brb3.1730

Neto MLR et al (2020) When health professionals look death in the eye: the mental health of professionals who deal daily with 2019 coronavirus outbreak. Psychiatry Res 288:e112972. https://doi. org/10.1016/j.psychres.2020.112972

Nolte S, Elsworth GR (2014) Factorial invariance. In: Michalos AC (ed) Encyclopedia of quality of life and well-being research. Springer, Dordrecht, p 311. https://doi.org/10.1007/978-94-007-0753-5

Occupational Safety and Health Administration (2020) Worker Expose risk to COVID-19 [PDF]. https://www.osha.gov/Publications/ OSHA3993.pdf Accessed 9 Dec 2020

Ornell F, Halpern SC, Kessler FHP, Narvaez JCM (2020) The impact of the COVID-19 pandemic on the mental health of healthcare professionals. Cad Saude Publica 36:e0063520. https://doi. org/10.1590/0102-311X00063520 
Pappa S, Ntella V, Giannakas T, Giannakoulis VG, Papoutsi E, Katsaounou P (2020) Prevalence of depression, anxiety, and insomnia among healthcare workers during the covid-19 pandemic: a systematic review and meta-analysis. Brain Behav Immun 88:901907. https://doi.org/10.1016/j.bbi.2020.05.026

Peditto M, Scapellato S, Marciano A, Costa P, Oteri G (2020) Dentistry during the COVID-19 epidemic: an italian workflow for the management of dental practice. Int J Environ Res Public Health 17:3325. https://doi.org/10.3390/ijerph17093325

Pfefferbaum B, North CS (2020) Mental health and the Covid-19 pandemic. N Engl J Med 383:510-512. https://doi.org/10.1056/ NEJMp2008017

Pierce M, McManus S, Jessop C et al (2020) Says who? The significance of sampling in mental health surveys during COVID-19. Lancet Psychiatry 7:567-568. https://doi.org/10.1016/S2215 -0366(20)30237-6

Rajkumar RP (2020) COVID-19 and mental health: a review of the existing literature. Asian J Psychatry 52:102066. https://doi. org/10.1016/j.ajp.2020.102066

Rinaldi L, Locati F, Parolin L, Girelli L (2017) Distancing the present self from the past and the future: psychological distance in anxiety and depression. Q J Exp Psychol 70:1106-1113. https://doi. org/10.1080/17470218.2016.1271443

Salazar de Pablo G et al (2020) Impact of coronavirus syndromes on physical and mental health of health care workers: systematic review and meta-analysis. J Affect Disord 275:48-57. https://doi. org/10.1016/j.jad.2020.06.022

Santamaría MD, Ozamiz-Etxebarria N, Rodríguez IR, Alboniga-Mayor JJ, Gorrotxategi MP (2020) Psychological impact of COVID-19 on a sample of Spanish health professionals. Rev de Psiquiatr y Salud Ment. https://doi.org/10.1016/j.rpsm.2020.05.004

Sethi BA, Sethi A, Ali S, Aamir HS (2020) Impact of coronavirus disease (COVID-19) pandemic on health professionals. Pak J Med Sci 36:S6-S11. https://doi.org/10.12669/pjms.36.COVID 19-S4.2779

\section{Authors and Affiliations}

\section{Juliana Alvares Duarte Bonini Campos ${ }^{1}$ (D) Bianca Gonzalez Martins ${ }^{1}$ (D) - Lucas Arrais Campos $^{2,5}$ (D) Filomena de Fátima Valadão-Dias ${ }^{3}$ (D) . João Marôco ${ }^{4}$ (D)}

\author{
Bianca Gonzalez Martins \\ bianca.g.martins@unesp.br \\ Lucas Arrais Campos \\ lucas.campos@unesp.br \\ Filomena de Fátima Valadão-Dias \\ valadaodiasfilomena@gmail.com \\ João Marôco \\ jpmaroco@ispa.pt
}

1 School of Pharmaceutical Sciences, São Paulo State University (UNESP), Rodovia Araraquara Jaú, Km 01, Araraquara, São Paulo 14800-903, Brazil
Shechter A et al (2020) Psychological distress, coping behaviors, and preferences for support among New York healthcare workers during the COVID-19 pandemic. Gen Hosp Psychiatry 66:1-8. https ://doi.org/10.1016/j.genhosppsych.2020.06.007

Usher K, Durkin J, Bhullar N (2020) The COVID-19 pandemic and mental health impacts. Int J Ment Health Nurs 29:315-318. https ://doi.org/10.1111/inm.12726

Vignola RCB, Tucci AM (2014) Adaptation and validation of the depression, anxiety and stress scale (DASS) to Brazilian Portuguese. J Affec Disord 155:104-109. https://doi.org/10.1016/j. jad.2013.10.031

Vizheh M, Qorbani M, Arzaghi SM, Muhidin S, Javanmard Z, Esmaeili M (2020) The mental health of healthcare workers in the COVID19 pandemic: A systematic review. J Diabetes Metab Disord 26:112. https://doi.org/10.1007/s40200-020-00643-9

Wang C, Pan R, Wan X, Tan Y, Xu L, Ho CS, Ho RC (2020) Immediate psychological responses and associated factors during the initial stage of the 2019 coronavirus disease (COVID-19) epidemic among the general population in China. Int J Environ Res Public Health 17:1729. https://doi.org/10.3390/ijerph17051729

Weiss DS, Marmar CR (1997) The impact of event scale—revised. In: Wilson JP, Keane TM (eds) Assessing psychological trauma and PTSD. Guilford Press, New York, pp 399-411

Zhang WR et al (2020) Mental health and psychosocial problems of medical health workers during the COVID-19 epidemic in China. Psychother Psychosom 89:242-250. https://doi. org/10.1159/000507639

Publisher's Note Springer Nature remains neutral with regard to jurisdictional claims in published maps and institutional affiliations.
2 School of Dentistry, São Paulo State University (UNESP), Campus (Araraquara), Rua Humaitá, 1680, Centro, Araraquara, SP 14801-140, Brazil

3 PsiRelacional (Relational Psychoanalysis Association), Consultório Almirante Reis, Almirante Reis, $n^{\circ}$ 238/1 ${ }^{\circ}$ Direito, 1000-166 Lisbon, Portugal

4 William James Center for Research (WJCR), University Institute of Psychological, Social, and Life Sciences (ISPA), Rua Jardim Do Tabaco, n³4, 1149-041 Lisbon, Portugal

5 Faculty of Medicine and Health Technology, Tampere University, Finn-Medi 2, FI-33520 Tampere, Finland 